\title{
Les mutations des systèmes productifs en France
}

\section{Raymond Woessner}

\section{OpenEdition}

\section{Journals}

Electronic version

URL: http://journals.openedition.org/rge/5186

DOI: $10.4000 /$ rge.5186

ISSN: 2108-6478

\section{Publisher}

Association des géographes de l'Est

Printed version

Date of publication: 15 October 2014

ISSN: 0035-3213

\section{Electronic reference}

Raymond Woessner, "Les mutations des systèmes productifs en France », Revue Géographique de I'Est [Online], vol. 54 / 1-2 | 2014, Online since 01 November 2014, connection on 23 September 2020. URL : http://journals.openedition.org/rge/5186 ; DOI : https://doi.org/10.4000/rge.5186

This text was automatically generated on 23 September 2020

Tous droits réservés 


\title{
Les mutations des systèmes productifs en France
}

\author{
Raymond Woessner
}

L'intitulé «France: mutations des systèmes productifs» est une nouvelle question posée aux concours de l'agrégation en 2014 et du CAPES en 2015. Dans sa lettre d'accompagnement d'octobre 2013, le jury d'agrégation avait précisé qu'il ne s'agit pas seulement d'évoquer l'industrie manufacturière «mais aussi les services marchands » et l'agriculture. Cette lettre met également en avant les différents secteurs d'activité, les approches multiscalaires, les acteurs publics et les entreprises, tout comme les systèmes d'aides publiques. Le champ ainsi ouvert est immense si, dans une perspective monographique, l'on prend en compte d'innombrables secteurs d'activité et territoires. Chaque cas peut être expliqué dans sa genèse et dans son évolution. Et en même temps, au-delà de son originalité, il s'inscrit dans une loi générale. On peut en effet admettre qu'un système productif naît, grandit, mûrit, décline ou se renouvelle tant en fonction de ses ressources propres que du contexte qui l'englobe. C'est ainsi qu'un cas de figure donné prendra sens. 
Figure 1 : Les Trente Glorieuses puis la mondialisation

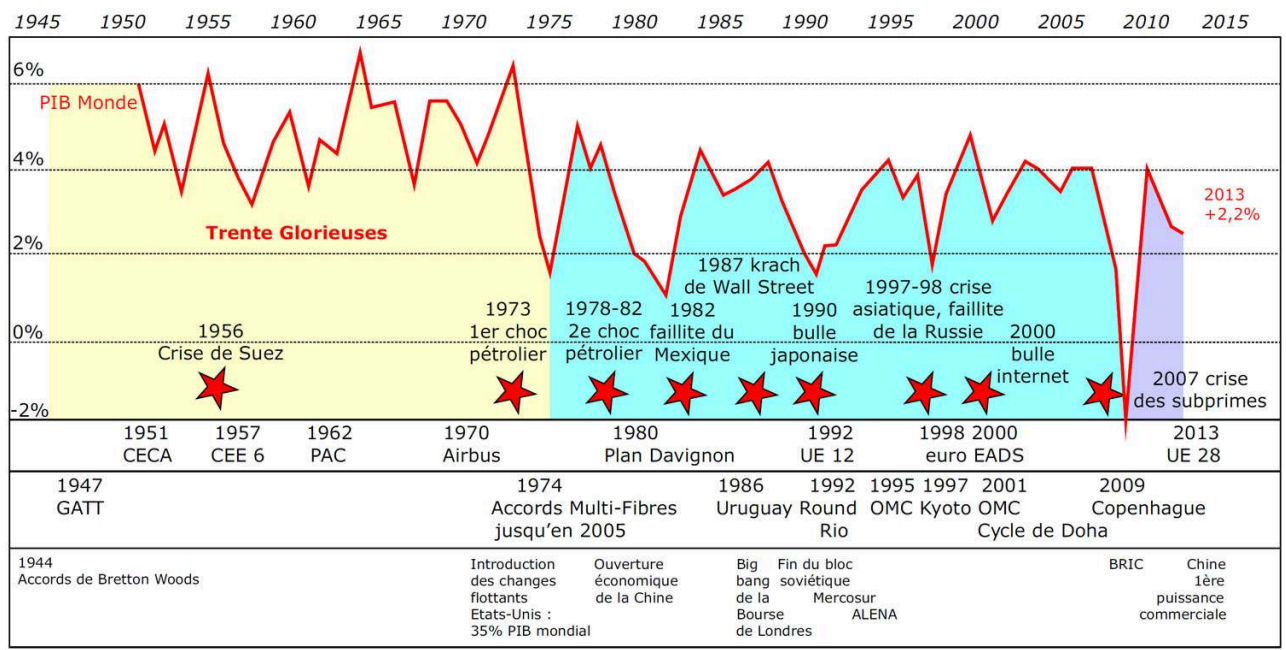

Figure 2 : Les Trente Glorieuses puis la mondialisation en France

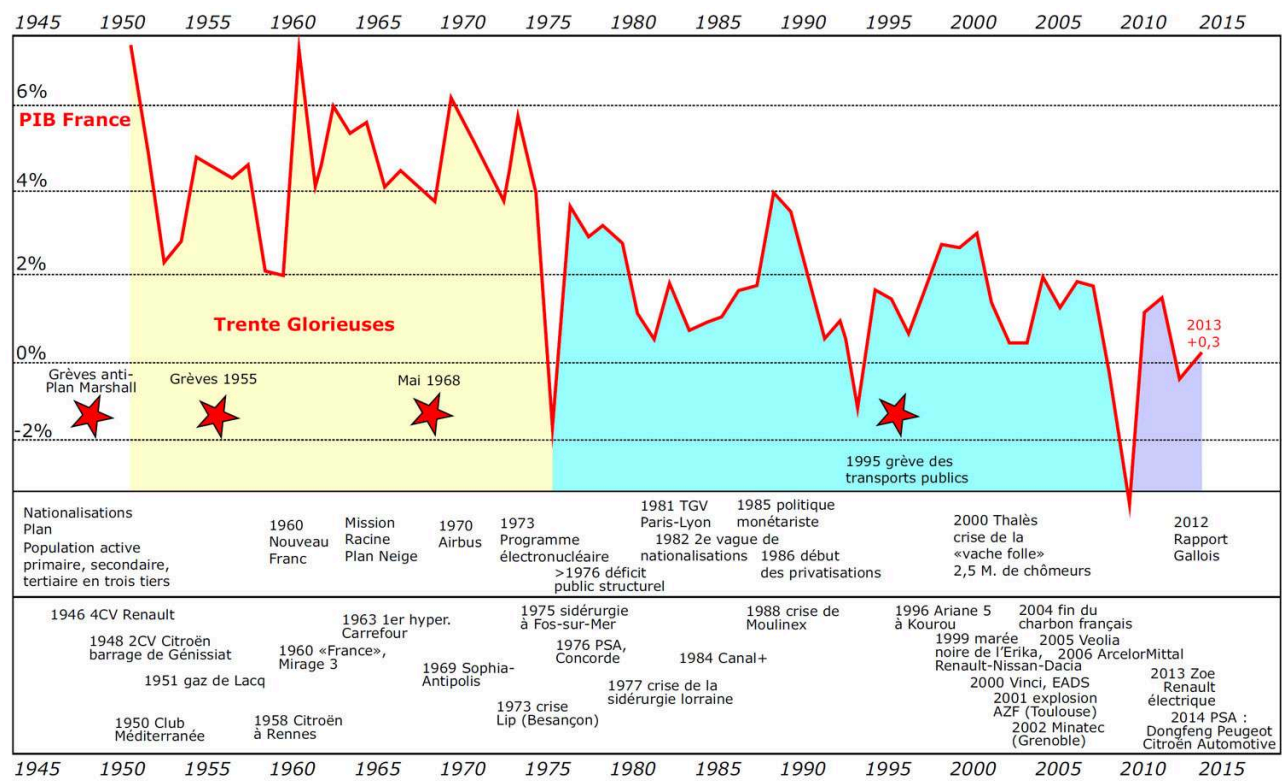

2 Depuis plusieurs décennies, la mondialisation libérale et le capitalisme financier ont remis l'ensemble du patrimoine économique français en cause (Figures 1 et 2). La robustesse d'une implantation se joue désormais à trois échelles. Localement, les facteurs microéconomiques renvoient à la capacité du management de l'entreprise à prendre les bonnes décisions. Globalement, les facteurs macroénomiques concernent la compétitivité de la plate-forme France. Entre ces deux échelles, les territoires peuvent prendre des dimensions variées, et c'est à leur niveau que les enjeux géographiques prennent sens. En effet, dès avant 1914, la littérature des économistes spatiaux anglosaxons et même la production de Vidal de La Blache se sont interrogées sur les apports des compétences, des ressources, des aménités, des externalités fournies par un territoire et qui ne figurent ni dans le bilan comptable d'une entreprise, ni dans la comptabilité nationale. La célèbre phrase d'Alfred Marshall, « les secrets de l'industrie sont dans l'air que l'on respire ", interpelle les limites de la pensée quantitative et des aspects purement rationnels. Elle invite à réfléchir à l'enrobage qualitatif des districts 
productifs. Longtemps, on ne s'y est guère intéressé parce que la modernité fordiste semblait apte à garantir la croissance économique et, partant, la fabrication territoriale (Figure 3). Ce n'est qu'à partir des années 1980 que les travaux sur les Systèmes Productifs Locaux (SPL) portant sur la Troisième Italie (Beccatini, 1987) ou sur le Jura suisse (Maillat, 1993) ont soufflé sur cette braise dont la DATAR s'était rapidement emparée pour proposer des solutions en vue du renouveau du tissu productif français, avec la promotion des districts, des grappes et autres pôles de compétitivité (DATAR, 2004).

Figure 3 : La Rhodia à Besançon

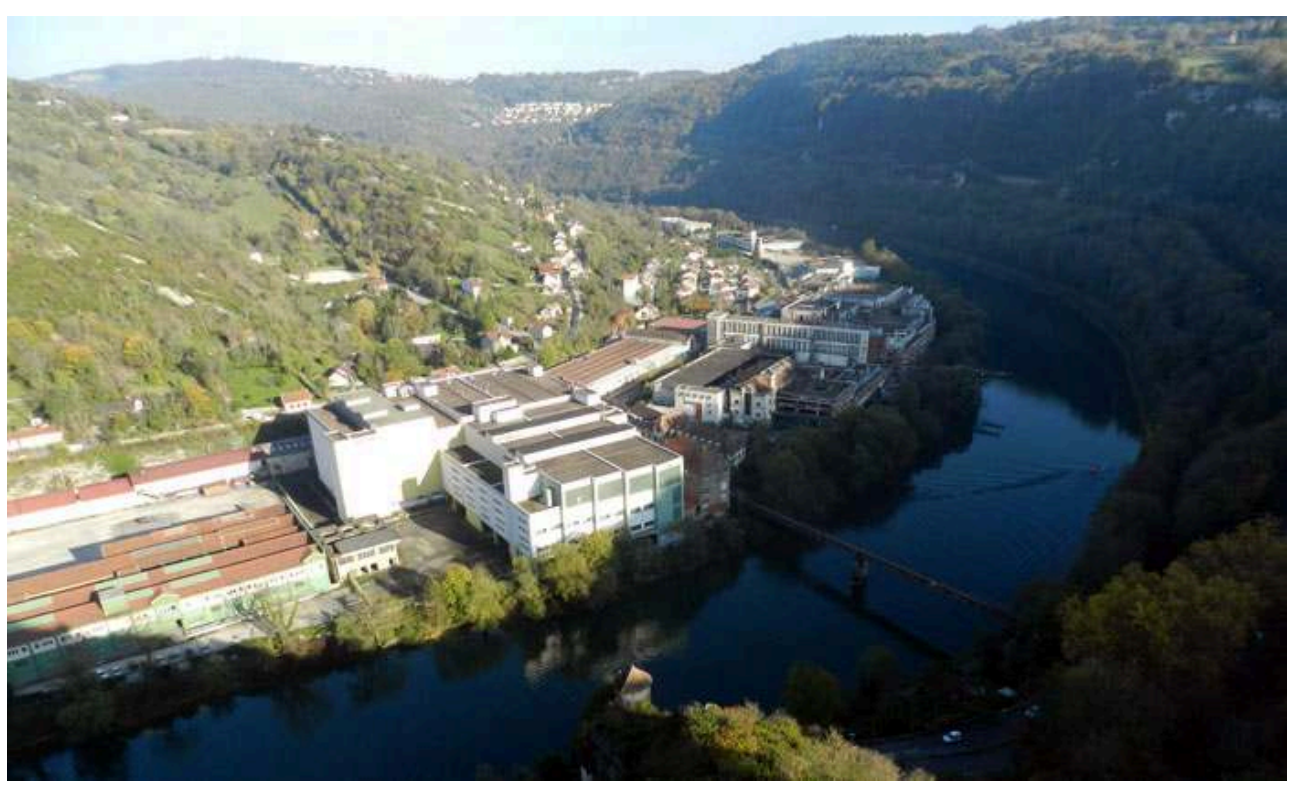

Photo Raymond Woessner 31 octobre 2014

3 L'usien Rhodiacéta commence à fabriquer du fil polyester à partir de 1952, sur le site d'une ancienne usine de soie artificielle ouverte en 1892. En 1967, la Rhodia est le premier employeur bisontin avec 3000 salariés, mais elle connait alors une grève très dure. Elle ferme en 1982. Commence alors un enfrichement qui est toujours d'actualité, dans un site de grande qualité au bord du Doubs et juste en amont des portes de la ville. Depuis, la municipalité de Besançon essaie d'acquérir le site pour le transformer en quartier résidentiel, mais les procédures judiciaires peinent à se terminer. A proximité (hors photo) est déjà apparu la Rodia, une salle de spectacles et de concerts.

4 Nous voici donc face au problème de l'innovation, que l'on perçoit souvent comme étant technologique. Or celle-ci ne peut « percer » que si l'acceptabilité sociale, codifiée par une réglementation adaptée, le permet. C'est ainsi que sont convoqués les mentalités, les représentations et les désirs. Il appartient au monde politique de permettre leur concrétisation, alors même que divers lobbies vont chercher à pérenniser des rentes que l'innovation menace. Si cet obstacle est surmonté, de nouveaux territoires peuvent émerger, sur des bases infiniment variées. Ainsi, l'innovation est plurielle dans le sens où elle concerne des jeux d'acteurs de statut et d'importance diverses.

5 Avec bonheur, l'intitulé de la question ouvre deux pistes de recherche au moins, à propos des « mutations » et des « systèmes productifs ». 
6 Les « mutations » contiennent un fort potentiel de réflexion. On voit bien que la France peine à s'adapter à la mondialisation. Il apparaît difficile de se remettre en cause lorsqu'on est une vieille civilisation qui a exporté ses produits et ses valeurs dans le monde. Mais face à cette variable forçante, il faut muter ou même muer, sortir de sa carapace pour affronter un monde concurrentiel. La rupture, la bifurcation, le changement, la mutation, l'innovation, la créativité sont autant de termes qui témoignent d'une situation de crise lorsque la trajectoire du système est à revoir. Ainsi, un système territorial peut-il être condamné au dépérissement ? Ou bien saura-t-il bifurquer vers un nouveau système ? Dans ce cas, la rupture est-elle lente ou brutale? En voit-on des signes annonciateurs, des marqueurs d'évolution? Et quelle sera l'importance des rétroactions? Aujourd'hui, nombreuses sont les déclarations de chefs d'entreprise ou les publications de presse qui évoquent « l'ADN » d'une firme, à moins qu'on ne préfère "changer de logiciel » ou créer un "nouvel écosystème ». Tant qu'à faire, autant le proclamer en anglais avec "back to the race" ou "cash is king", comme l'a fait Carlos Tavares en prenant les commandes de Dongfeng Peugeot Citroën Automotive (DPCA), en avril 2014. Ces métaphores disent que somme toute il vaut mieux bifurquer dans un monde que l'on sait appréhender à travers ses interactions, et que l'on peut envisager une "mutation» en restant fidèle à une forme de capital culturel qui s'est accumulé depuis le début d'une histoire donnée. Au contraire, l'émergence, une notion qui reste sujette à discussion chez les systémistes, serait plus brutale. On peut admettre qu'elle se produit lorsqu'un nouveau jeu d'acteurs se met en place, et pour le dire brutalement, lorsqu'une coalition de projet remplace une clique de rentiers.

7 Les «systèmes productifs » sont d'abord des systèmes, que l'on peut classer en deux catégories, simple ou complexe. Un système simple fonctionne sur la base d'un nombre limité d'éléments et de relations. La principale caractéristique d'un système simple est donc celle de la prévisibilité des événements à venir. À une échelle géographique donné, à condition d'organiser une stricte régulation sociale et politique, et de ne pas trop dépendre d'une variable forçante extérieure, il est facile de se prémunir des accidents éventuels. Dans ce cas, le territoire fonctionne comme un cocon spatiotemporel. On songe à la France des Trente Glorieuses (1945-1975), lorsque l'INSEE et le Commissariat au Plan pouvaient mettre le pays en équations. La modification d'une variable altérait alors tout le système de manière prévisible, et cela sans grand risque d'erreur. On songe également à la première édition dans les années 1960 des Rouages de l'économie nationale. Jean-Marie Albertini y décrivait alors avec brio le circuit économique de la comptabilité nationale.

8 Plus de quarante ans plus tard, le même auteur a proposé une nouvelle édition de son ouvrage (Albertini, 2008). A présent, il évoque la mondialisation, le développement durable et le capitalisme sans éthique. De fait, il ajoute de la complexité à son analyse. Le système complexe mobilise davantage d'éléments et de relations que le système simple. Il se compose d'éléments nombreux et différenciés qui interagissent de manière non triviale (interactions non-linéaires, boucles de rétroaction, etc.) dans une apparence de désordre - en fait, dans un ordre que l'on a du mal à saisir. Il se caractérise par l'apparition de propriétés nouvelles et par une dynamique de fonctionnement global difficilement prédictible. "Un système complexe typique a un comportement holistique qui rend vaine toute tentative d'analyse par une découpe en sous-systèmes plus simples » (Rolland-May C., 2005). On peut donc évoquer un système 
complexe lorsqu'il y a incertitude pour les évolutions à venir. Celles-ci sont souvent difficiles à déceler. Dès 1903, le mathématicien Henri Poincaré, dans "Science et méthode", soulignait que de petites différences dans des conditions initiales en produisent de très grandes lorsqu'on observe le phénomène final. Tel est le monde d'aujourd'hui, soumis à des événements nombreux, inattendus et susceptibles de remettre en cause mainte construction économique ou politique. Les logiques financières court-termistes ne font qu'attiser ces incertitudes (Tétreau, 2005).

Le pluriel de "systèmes productifs " peut renvoyer à deux interprétations au moins. Les logiques de grappes introduisent la notion de territorialité. Pour une aire donnée, l'analyse géographique prend en compte les activités présentes. Elle s'intéresse aux relations fondées sur l'antagonisme de la concurrence et de la complémentarité. Alfred Marshall (1842-1942) et Joseph Schumpeter (1883-1950) ont été des pionniers en s'intéressant aux ressources spécifiques d'un territoire donné. Les externalités, c'est-àdire l'environnement dans lequel une entreprise évolue, expliquent alors la compétitivité d'un territoire. Alfred Chandler (1918-2007) a finalisé le raisonnement en évoquant le cluster, lorsqu'un avantage compétitif résulte d'une agrégation d'entreprises qui tire les services innovants avec elles. Par ailleurs, le pluriel de «systèmes productifs" est également justifié par l'approche multiscalaire. Il est possible de raisonner à l'échelle du monde, de l'Union européenne, de la France ou de ses composantes régionales et locales. Cependant, dans un pays unitaire, de tradition centralisatrice, l'État reste un acteur déterminant qui crée une forme d'homogénéité sur l'ensemble de son territoire.

10 Mais quelle est la clé qui permet d'expliquer «la création destructrice» contemporaine, pour reprendre une expression de Schumpeter ? Ce sont moins les éléments que leurs relations qui font sens. Il est banal de dire qu'autrefois l'usine faisait le territoire et qu'aujourd'hui c'est l'inverse. L'entreprise est plongée - ou pas! dans un tissu relationnel où elle trouvera les facteurs de compétitivité qui lui sont nécessaires. Or, la France est organisée de manière pyramidale et verticale. A une échelle géographique donnée, les relations entre les acteurs fonctionnent mal, dans l'attente d'une prise de décision supérieure, alors même que l'entreprise a un besoin vital de connexion, d'information et de proximité. « Je suis descendu de l'avion à Dijon et il n'y avait personne pour m'accueillir ", avait déclaré le PDG de Hoover à la presse, lorsqu'on lui reprochait de s'être finalement délocalisé en Écosse au détriment de la Bourgogne. C'était en 1993, à une époque où les avions de ligne se posaient encore régulièrement à Dijon-Longvic.

11 Notons que l'internetisation apporte une forme d'ubiquité et d'instantanéité à la diffusion de l'information, qu'elle abolit le temps et l'espace, et cela toutes échelles confondues. Et pourtant, on voit se dégager des strates territoriales qui gagnent et d'autres qui perdent. Au premier rang, les métropoles jouent pleinement sur les logiques d'archipel. Elles se projettent au loin depuis la première mondialisation (Braudel, 1985). A présent, avec l'ouverture des frontières et les techniques adaptées, elles se détachent littéralement de leur susbtrat national, concentrent les activités profitables et innovantes, deviennent des métapoles (Ascher, 1995) en se s'implantant de manière discontinue hors de leurs murs. Le reste du territoire national, c'est-à-dire tout ce qui n'est pas connecté d'une manière ou d'une autre aux métropoles, est-il voué au déclin? Dans le cadre du paradigme de la mondialisation libérale, quels sont alors les facteurs qui peuvent encore y assurer le dynamisme économique ? Faut-il sortir du 
marasme par le haut (une production de biens et de services à forte valeur ajoutée assise sur une image de marque) ou par le bas (en brisant les coûts de production et le modèle social) ? Ou faut-il s'appuyer sur des émergences, mais sur quelles bases ?

Le présent numéro de la RGE invite à une lecture sectorielle du système productif français. Il s'intéresse d'abord au monde agricole avec les contributions de Sylvaine Fassier-Boulanger ( $\mathrm{ENeC}$, Paris Sorbonne) qui établit un tableau général de la situationa agricole, puis de Daniel Ricard (CERAMAC, Clermont-Ferrand) pour les mutations particulièrement complexes des filières laitières. Dalila Messaoudi (CEMOTEV, Guyancourt) mesure les dégâts relatifs provoqués par les délocalisations dans l'industrie manufacturière. Antoine Grandclément (TELEMME, Lyon) explique comment cette industrie manufacturière est désormais tirée en avant par les pôles de compétivité, avec une approche illustrative centrée sur la région PACA. Téva Meyer (IFG, Paris 8) expose la territorialisation d'un fleuron scientifique, technologique et industriel national, à savoir la filière nucléaire, avec un point sur la Bourgogne. Enfin, Patricia Lejoux (LET, Vaulx-en-Velin) croise le défi énergétique avec les questions soulevées par une mobilité croissante, notamment à travers la périurbanisation.

\section{BIBLIOGRAPHY}

Albertini J.-M., 2008, Les nouveaux rouages de l'économie, Ed. de l'Atelier, 2008, 336 p.

Ascher F., 1995, Métapolis. L'avenir des villes, Paris Odile Jacob, 352 p.

Beccatini A., 1987, Mercatore forze locali : il distretto industriale, Bologne, Il mulino.

Braudel F., 1985, La dynamique du capitalisme, Paris Arthaud, 121 p.

DATAR, 2004, La France, puissance industrielle, Paris La Documentation française, 127 p.

Maillat D., dir., 1993, Villes de l'Arc jurassien : quels enjeux pour le développement régional ?, Neuchâtel EDES, 140 p., extrait p. 25.

Rolland-May C., 2005, Frontières, limites, discontinuités, ruptures, mutations dans un système géographique complexe, dossier de demande de statut pour un groupe de recherche, Strasbourg, Université Louis Pasteur, cf. p.11.

Tétreau E., 2005, Analyste, au cœur de la folie financière, Paris Grasset, 278 p.

\section{AUTHOR}

\section{RAYMOND WOESSNER}

Professeur de géographie, Paris Sorbonne - 191, rue Saint-Jacques 75005 Paris raymond.woessner@wanadoo.fr 